\title{
AN ULTRA-LONG-PERIOD SEISMOGRAPH GALVANOMETER
}

\author{
By Francis E. Lehner
}

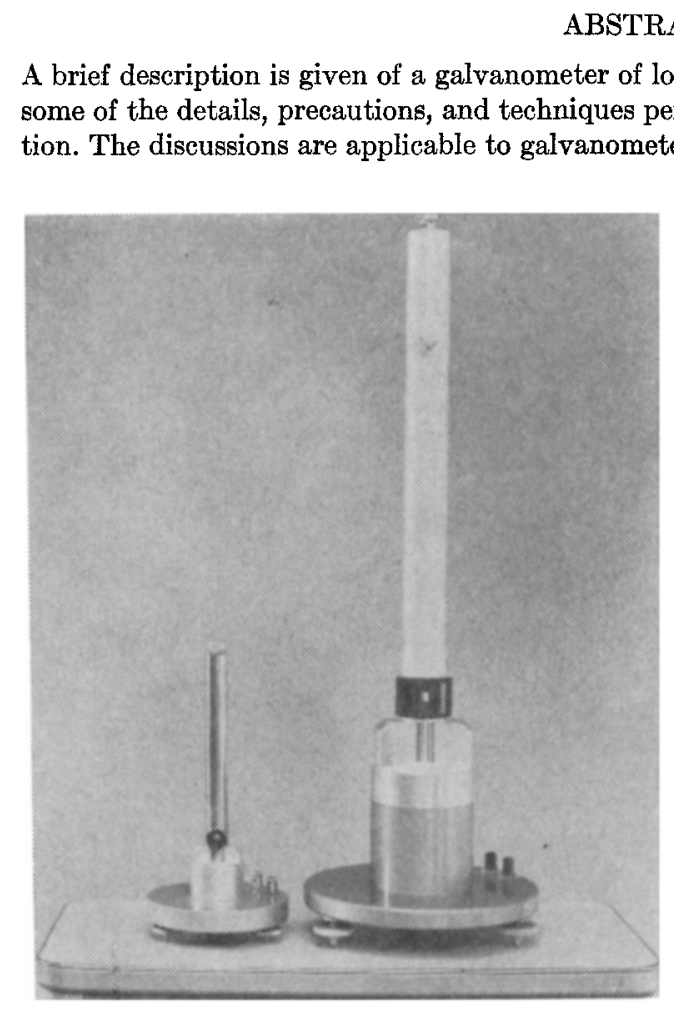

Fig. 1. A 90-sec. and a 480-sec. galvanometer

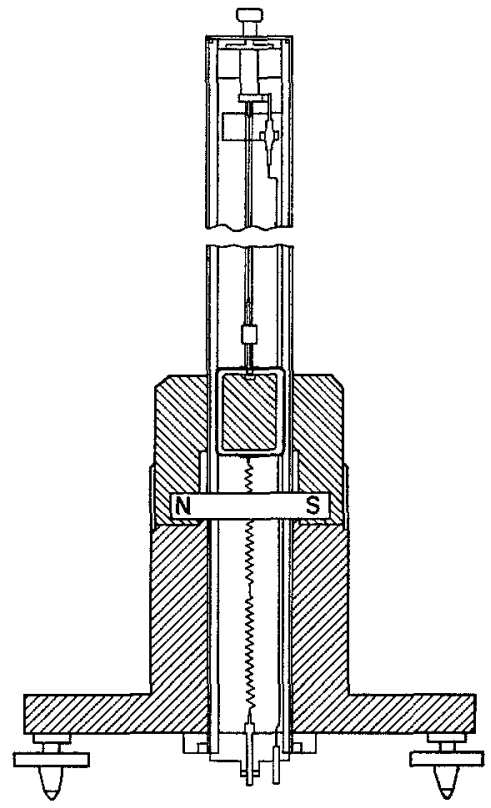

Fig. 2. Schematic section of 480 -sec. galvanometer

THE instrument described herein (figs. 1 and 2) is basically a scaled-up version of the 90 -second galvanometer of the author's design (1941) and currently in use in many long-period seismograph installations. Characteristics are as follows:

Period (corrected for air damping)..

Current sensitivity

External $R$ for critical damping

Instrument in vacuum approximately $10^{-4} \mathrm{~mm} \mathrm{Hg}$.

Coil resistance

Magnetic field strength..
480 seconds

$.4 \times 10^{-12} \mathrm{~A} / \mathrm{mm}$. at $1 \mathrm{M}$ $25,000 \mathrm{ohms}$

20,000 ohms 25 gauss

Figure 3 is a comparison of earthquake records from several combinations of seismometer and galvanometer periods. Emphasis of the longer-period waves by the system utilizing the 480-second galvanometer is clearly shown.

Manuscript received for publication May 13, 1959. 


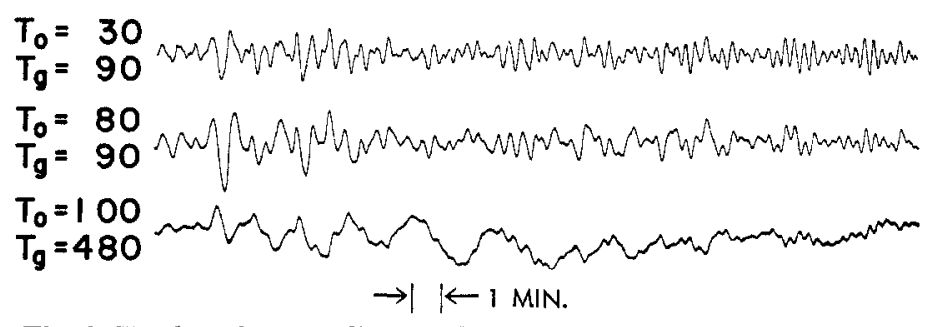

Fig. 3. Earthquake recordings with various instrument combinations. $3 \mathrm{~mm}$. equals $1 \mathrm{~min}$.

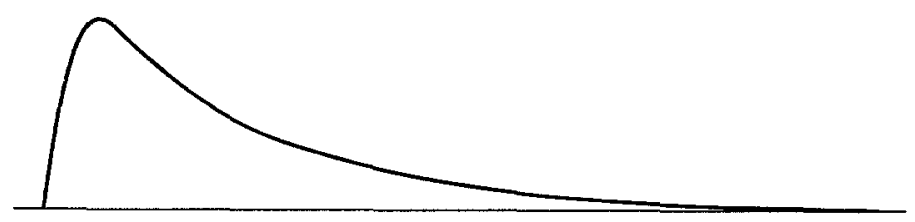

$$
\leftarrow 10 \text { MIN } \rightarrow-1
$$

Fig. 4. Air damping at atmospheric pressure.

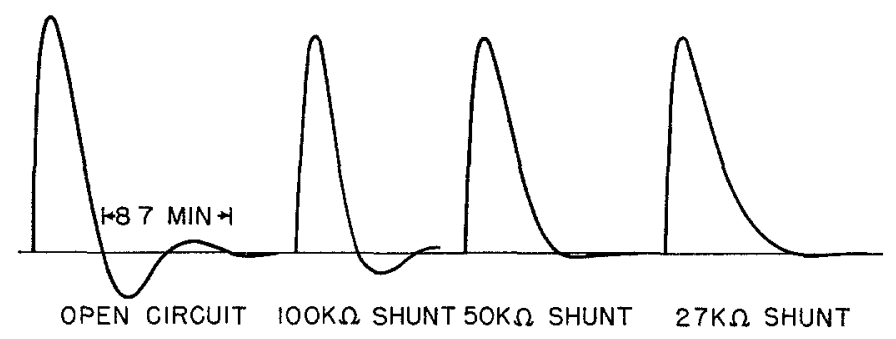

Fig. 5. Period and damping tests, instrument evacuated.

Because of the high air damping (fig. 4) it was necessary to make period and electrical damping tests with the instrument in an evacuated chamber. Since only a mechanical vacuum pump was used, the pressure attained was not below $10^{-4} \mathrm{~mm}$. of mercury and the residual gas was sufficient to cause a damping ratio of between 4 and 5 during the testing period. It was not possible, without pumping, to maintain a pressure sufficiently low to give damping less than critical for more than 24 hours, due to out-gassing of the component parts of the instrument. It is hoped that with repeated pumping at elevated temperature the chamber will eventually clean up sufficiently to maintain conditions at least as good as those in figure 5 . The instrument case is now being redesigned to make the galvanometer more adaptable to evacuated operation.

\section{Construction Details}

A formless coil of 2,400 turns of 0.001 in. diam. enameled wire and approximately 2 in. square is suspended in the magnetic gap by $24-\mathrm{K}$ gold ribbon having dimensions of 0.00012 by 0.003 in. and a torsion constant of 0.04 dyne $\mathrm{cm} / \mathrm{cm} / \mathrm{radian}$. The 
suspension is soldered to an elongated copper coil tab which carries the mirror. Electrical connection to the coil is through the top suspension and through a helix $6 \mathrm{in}$. long by $1 / 16 \mathrm{in}$. diam. wound from 24 in. of the same gold ribbon and soldered to a bottom coil tab. Coil weight is approximately 3 grams; breaking strength of the suspension is approximately 8 grams.

Torsion of the helix approaches that of the suspension. Period, calculated on the basis of the top suspension only, is 670 seconds.

Zero adjustment is accomplished by rotating the top suspension attachment, a coil clamp which also slacks the suspension provides for safe transport.

The magnetic field assembly consists of two iron pole pieces having faces concentric about a cylindrical iron core centered on the suspension axis. Gap between the core and pole faces is small as compared with the mean gap diameter, and gap length along the arc is great as compared with the maximum operating swing of the coil. Thus the coil is suspended in a zone of comparatively pure radial field, and influence on instrument behavior by magnetic impurities within the coil is minimized. To further assure symmetry in the field configuration the magnet proper is placed at some distance below the pole pieces, and flux carriers parallel to the axis of the gap connect it with the outer portion of the poles.

Wire used in the coil was washed twice while passing between poles of a magnet, to collect magnetic particles dislodged. Coil cements and solvents used, likewise, were kept in containers with magnetic collectors, and strict cleanliness was observed in handling materials and in assembling of the instrument.

With the exception of the gold suspensions the electrical circuit is completely of copper; gold-copper junctions are balanced on each side of the coil.

In testing and operating it was found necessary to provide a leak between the suspended system and the case in order to neutralize electrostatic charges. Otherwise, electrostatic forces gave erratic behavior and frequently complete instability. (While exaggerated here, such behavior is not peculiar to this instrument. Galvanometers of more ordinary periods behave similarly in locations having low humidity.)

This instrument was constructed in the instrument shop of the Seismological Laboratory, and its success is in large part due to the craftsmanship of Mr. Carl Holmstrom, Mr. David Downing, and Mr. Ivar Sedleniek. Valuable suggestions were made by Dr. Frank Press and by Dr. Hugo Benioff, whose research originally required such an instrument and initiated its design.

California Institute of Technology,

Pasadena, California.

(Division of Geological Sciences contribution no. 925.) 